\title{
Reconciliation of Object Interaction Models
}

\author{
George Spanoudakis and Hyoseob Kim \\ Department of Computing, \\ City University, \\ Northampton Square, London EC1V 0HB, UK \\ E-mail: gespan@soi.city.ac.uk, hkim69@soi.city.ac.uk
}

\begin{abstract}
This paper presents Reconciliation+, a tool-supported method which identifies overlaps between models of different object interactions expressed as UML sequence and/or collaboration diagrams, checks whether the overlapping elements of these models satisfy specific consistency rules, and guides developers in handling these inconsistencies. The method also keeps track of the decisions made and the actions taken in the process of managing inconsistencies.
\end{abstract}

\section{Introduction}

The design of software systems using the use-case driven approach [23] often results in the generation of multiple object interaction diagrams (i.e. sequence and/or collaboration diagrams). These diagrams are constructed to model specific interactions between the objects in a software system and the actors in its environment, which are required to deliver the functionality described by the particular use case that the diagram describes. These diagrams may be constructed independently by different designers, may advocate specific modelling angles and may reflect disparate perceptions of the system by these designers. As a result, they may have modelling inconsistencies.

Modelling inconsistencies occur when interaction diagrams incorporate messages which overlap that is they invoke operations with the same implementation - and model these messages in ways that violate specific consistency rules.

As an example, consider an object-oriented design model including the object interaction diagrams $\mathrm{I}_{1}$ and $I_{2}$ in Figures 4 and 5 . These diagrams show the interactions between the classes of a library system, which occur when the system is used to search for library items by keywords referring to the author and the title of an item, respectively. Assume also the following consistency rule:

CR1: If a message $m_{i}$ overlaps with a message $m_{j}$ then for every message $m_{k}$ dispatched by $m_{i}\left(m_{j}\right)$ there must exist a message $m_{w}$ dispatched by $m_{j}$ $\left(m_{i}\right)$ such that $m_{k}$ and $m_{w}$ overlap.

Given the class diagram of Figure 6, it may be reasonably assumed that the messages 7:actionPerformed(ActionEvent) in $\mathrm{I}_{1}$ and 10:actionPerformed(ActionEvent) in $\mathrm{I}_{2}$ overlap ${ }^{1}$. This is because both of them invoke the operation actionPerformed(e:ActionEvent) in the class DatabaseActionListener. Given this overlap, these two message violate CR1 since:

- 7:actionPerformed(ActionEvent) in $\mathrm{I}_{1}$ dispatches the message 8:getText() that does not overlap with any of the messages dispatched by 10:actionPerformed(ActionEvent), and

- 10:actionPerformed(ActionEvent) in $\mathrm{I}_{2}$ dispatches the messages 11:getData(), and 12:formulateQuery() which do not overlap with any of the messages dispatched by 7:actionPerformed(ActionEvent),

This paper describes a tool-supported method, called "Reconciliation+", that we have developed to support the detection of overlaps, checking of consistency rules and handling of violations of these rules (i.e., inconsistencies) in models of software interactions expressed as sequence (or collaboration) diagrams in UML [9]. Reconciliation+ has been developed as an extension of a method developed by Spanoudakis and Finkelstein to reconcile object-oriented models of system structures [14]. The newly developed extension:

(i) incorporates a flexible matching algorithm that detects overlaps between messages which may be not identically modelled in diagrams (e.g.

\footnotetext{
1 In this paper, we refer to messages by putting a number indicating the order of their dispatch in the interaction diagram they belong to before their signatures. This number uniquely identifies a message in a diagram but is not taken into account in any of the computations described in the paper.
} 
messages with different signatures and messages sent to instances of different classes),

(ii) allows the specification of a wide-range of consistency rules, checks against overlapping messages and allows developers to alter existing consistency rules or add new ones,

(iii) incorporates alternative ways of handling the detected violations of the rules and allows developers to alter them or add new ways of habdling, and

(iv) guides (as opposed to enforcing) developers in selecting which rules to check and how to handle their violations.

The process of reconciling interaction models is driven by the enactment of an explicit process model incorporated by the method.

The rest of the paper is structured as follows. Section 2 presents an overview of Reconciliation+ and the prototype toolkit we have developed to support it. Section 3 describes the specification and enactment of the process model of the method. Section 4 describes the algorithm for detecting overlapping messages in different interaction diagrams. Section 5 describes the specification of consistency rules and the mechanism for detecting violations of these rules. Section 6 describes the scheme for specifying and executing different ways of handling inconsistencies. Section 7 overviews related work and, finally, Section 8 summarises the method and outlines the current and future work on it.

\section{Overview of Reconciliation+}

Reconciliation+ is supported by a toolkit whose general architecture is shown in Figure 1. This toolkit incorporates a tool that detects overlaps between object interaction models and an engine which enacts a process model that drives the process of reconciling interaction diagrams. This process model specifies:

(i) The tool that may be invoked to identify overlaps in interaction models and the conditions under which this tool may be activated.

(ii) The consistency rules that can be checked against interaction models and the conditions under which these rules may be checked to detect inconsistencies. The consistency rules are expressed as queries. In this approach, the queries retrieve the model elements that violate the conditions that the rules require elements to satisfy and therefore violate the rules. (iii) Different ways of handling inconsistencies (specified by inconsistency handling actions) and the conditions under which each of these ways may be applied.

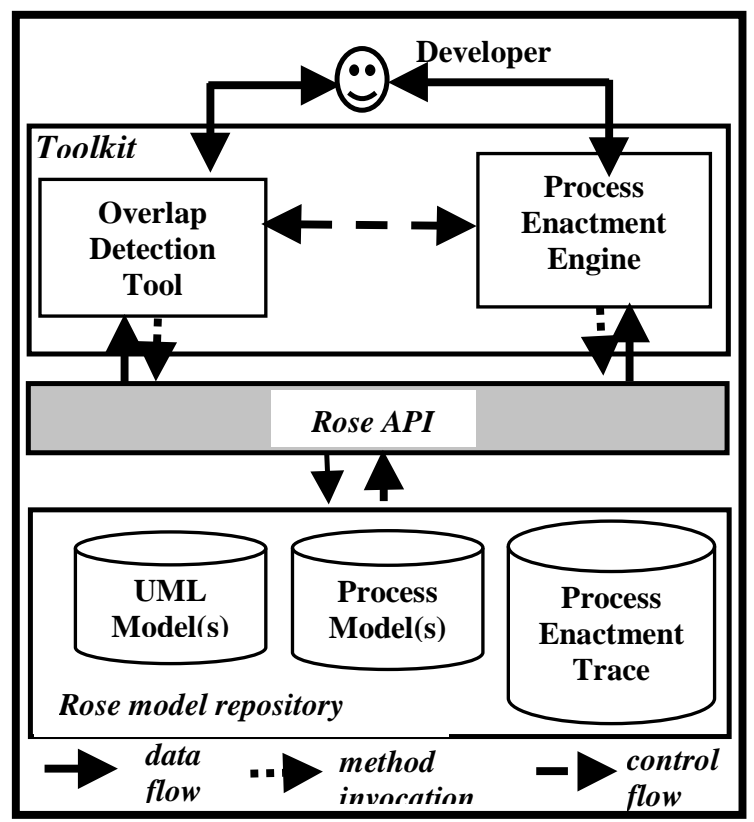

Figure 1: Architecture of the Reconciliation+ toolkit

The process model of the method is enacted by a process enactment engine. This engine interprets the process model, presents the available options to the developers, acts according to the option selected (or other instructions given by them), and keeps a trace of the enacted process.

The toolkit of the method has been implemented on the top of Rational Rose (a CASE tool supporting UML) using its API. The models to be reconciled are stored as collections of UML class models and sequence diagrams in a model repository managed by Rose. The process model of the method and the traces of the enacted processes are also represented as UML class models in the Rose repository.

\section{Specification and enactment of Reconciliation+ processes}

\subsection{The process meta-model}

The process model of Reconciliation+ is specified in UML as an instance of an extended version of the NATURE process meta-model [11,13]. A specification of this meta-model in UML is shown in Figure 2. 
According to the Nature meta-model (see non-grey classes in Figure 2) a process is described as a graph of contexts. A context represents the decision to pursue a specific goal (intention) in a given situation. A situation is a condition over the state of the software model being manipulated by the process. Contexts are distinguished into:

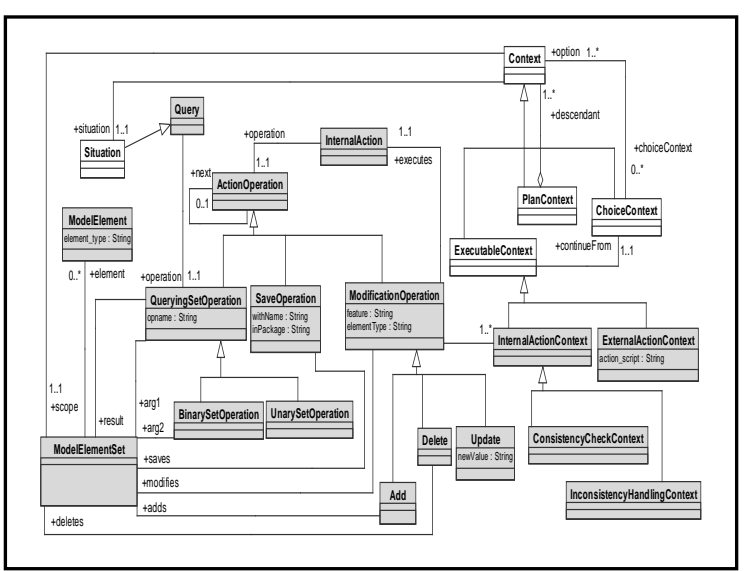

Figure 2: Process Meta-Model in UML

- executable contexts - these are contexts whose intentions that can be directly pursued by taking an action which changes the state of the software model

- plan contexts - these are contexts whose intentions are decomposed into a set of sub-goals which have to be pursued in a specific order

- choice contexts - these are contexts whose intentions are refined into one or more alternative goals and can therefore be satisfied by pursuing any of these goals

To be able to specify the process of Reconciliation+, we had to extend the Nature process meta-model in order to establish: (1) a scheme for specifying situations for processes which are enacted upon UML models, and (2) a scheme for specifying the types of actions required for detecting and handling inconsistencies. These extensions were necessary since the Nature metamodel does not determine how to specify context situations and actions. The extensions introduced are described in the following.

\subsection{Specification of situations}

In the extended meta-model, a situation is a query that is specified as an ordered sequence of querying set operations. As shown in Figure 2, a querying set operation can be: (i) a UnarySetOperation - These operations can be used to retrieve: (a) sets of elements (or primitive data values) from a UML model which are associated with a model element $e$ via the different kinds of associations or attributes defined by the UML meta-model for the type of $e$, or (b) the elements of a set which satisfy a specific condition (SelectOperation). A unary set operation is applied to each of the elements of its argl set and returns the union of the elements retrieved for each of them in its result set.

The extended meta-model defines a taxonomy of unary set operations for all the different types of model elements which are defined in the UML meta-model. Figure 3 shows a part of this taxonomy which includes the operations available for retrieving model elements and data values associated with UML classes. As an example, consider the operation GetAssociations in this taxonomy. This operation retrieves the associations which relate the classes in its $\arg 1$ set with other classes, creates instances of the process meta-model class ModelElement to represent them, and inserts these elements into its $\arg 2$ set (by associating the newly created model elements with this set - see the association end element in Figure 2).

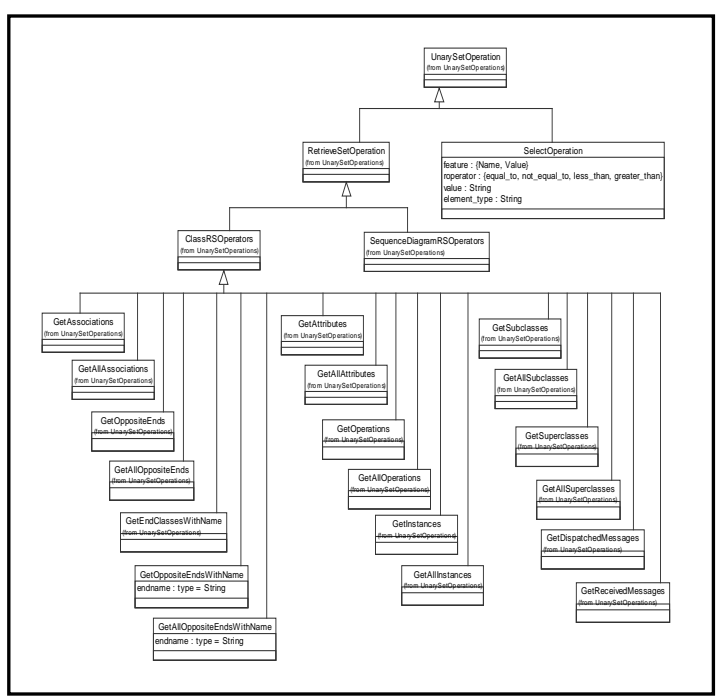

Figure 3: Part of the taxonomy of unary set operations

(ii) a BinarySetOperation - These operations realise the operations of set union, intersection and difference. Binary set operations are applied to the sets that constitute their arg1 and $\arg 2$ sets and generate a result set according to the 
standard definitions of their underlying mathematical operations.

The operations of a situation are executed in the order in which they appear in it. An operation may take as an argument any of the sets generated by an operation appearing before it in a situation. A situation is considered to be satisfied if the result set of the last of its operations is not empty. Examples of situations specified according to this meta-model are given in Section 6 below.

\subsection{Specification of actions}

Actions are defined for executable contexts and can be specified either as executable script file names (see attribute action_script of meta-class ExternalActionContext in Figure 2), or as instances of the meta-class InternalAction. An executable context may be an ExternalActionContext or an InternalActionContext depending on whether it includes an action of the former or the latter kind.

External actions are used to invoke tools (e.g. the tool that detects overlaps). Internal actions are used to specify consistency rules (in the operational form that we discuss in Section 5), and ways of handling inconsistencies.

Similarly to situations, internal actions are specified as sequences of action operations (see meta-class ActionOperation in Figure 2). The taxomomy of the different types of action operations is shown in Figure 2. As shown in this figure, an action operation may be a:

(a) querying set operation (see Section 3.2).

(b) model modification operation - This is an operation which modifies (i.e., adds, deletes or updates) elements of the retrieved sets (see meta-class ModificationOperation in Figure 2).

(c) save operation - This is an operation which saves retrieved sets of elements in the process enactment trace (see meta-class SaveOperation in Figure 2).

Sequences of internal action operations are interpreted and executed by the process enactment engine similarly to the set querying operations of situations. Examples of internal actions used to detect, and handle inconsistencies are given in Sections 5 and 6, respectively.

\subsection{Process enactment}

The Reconciliation+ process model is enacted by an engine which functions as a model interpreter [13].
The full algorithm implemented by the process enactment engine of the method is beyond the scope of this paper and may be found in [22]. In this section, we give only an overview of the enactment of process models in Reconciliation+.

The enactment of a model starts from the single root choice context that every process model must have. The situation of this context (and any other context that is encountered as the enactment engine traverses the process model) is evaluated by executing its set querying operations. If the set which results from the execution of the last of these operations (called "situation set") is not empty, the situation of the context is satisfied. If a situation is satisfied, the enactment engine generates $\mathrm{N}$ different possible decisions from its context where $\mathrm{N}$ is the number of the elements of its evaluated situation set.

\section{A decision is a pair where \\ - context ${ }_{i}$ is the context whose situation is satisfied, and \\ - situation_set_element ${ }_{j}$ is an element of the situation set of context . $_{\text {. }}$.}

The developer may select one of these decisions, ask for tactical guidance or terminate the process. If a decision <context, situation_set_element > is selected, the decision is recorded in the process trace (see Figure 1). Subsequently:

- If context is a choice context the enactment engine: (1) retrieves the option contexts associated with it, (2) inserts the situation_set_element in the argl set of the initial querying set operations of each of these contexts, (3) evaluates the situation of each of these contexts, (4) generates the possible decisions for each of these contexts, and (5) prompts the developer to make a new selection.

- If context $t_{i}$ is an external action context the enactment engine executes the file specified by its attribute action_script and continues the enactment of the process model from the context associated with context $t_{i}$ via the end continueFrom (see Figure 2).

- If context $t_{i}$ is an internal action context the enactment engine executes the sequence of the operations in its internal action and continues the 
enactment of the process model as in the case of external action contexts.

Plan contexts are not used in the current process model of the method and are not supported in the current implementation. In cases where the developer asks for tactical guidance, the enactment engine identifies the decision before the last decision recorded in the process trace and resumes execution from the context of it. The developer may abort the execution of the process model at any point.

\section{Detection of overlaps}

\subsection{Basic algorithmic formulation}

The detection of overlaps between sequence diagrams is formulated as an instance of the weighted bipartite graph matching problem [10]. Assuming a pair of sequence diagrams modelling two object interactions $I_{i}$ and $I_{j}$, we construct an interaction overlap graph:

$$
\operatorname{IOG}\left(\mathrm{I}_{\mathrm{i}}, \mathrm{I}_{\mathrm{j}}\right)=\left(\mathrm{V}_{\mathrm{i}} \cup \mathrm{V}_{\mathrm{j}}, \mathrm{E}\left(\mathrm{V}_{\mathrm{i}}, \mathrm{V}_{\mathrm{j}}\right)\right)
$$

This graph has two sets of vertices $V_{i}$ and $V_{j}$. Assuming (without loss of generality) that $I_{i}$ has more messages than $I_{j}, V_{i}$ and $V_{j}$ are defined as:

$$
\mathrm{V}_{\mathrm{i}} \equiv \operatorname{Messages}\left(\mathrm{I}_{\mathrm{i}}\right) \text { and } \mathrm{V}_{\mathrm{j}} \equiv \operatorname{Messages}\left(\mathrm{I}_{\mathrm{j}}\right) \cup \mathrm{DV}_{\mathrm{k}}
$$

where $\mathrm{DV}_{\mathrm{k}}$ is a set of $\mathrm{k}$ special vertices representing dummy messages $\left(\mathrm{k}=\left|\operatorname{Messages}\left(\mathrm{I}_{\mathrm{i}}\right)\right|\right.$ $\mid$ Messages $\left.\left(\mathrm{I}_{\mathrm{j}}\right) \mid\right)$. Vi and $\mathrm{Vi}$ are disjoint sets since each message must belong to only one interaction (see [9]). Thus, $\operatorname{IOG}\left(\mathrm{I}_{\mathrm{i}}, \mathrm{I}_{\mathrm{j}}\right)$ is a bipartite graph.

The set of the edges $\mathrm{E}\left(\mathrm{V}_{\mathrm{i}}, \mathrm{V}_{\mathrm{j}}\right)$ includes only edges which connect the messages of $I_{i}$ with the messages of $\mathrm{I}_{\mathrm{j}}$ :

$$
\begin{aligned}
\mathrm{E}\left(\mathrm{V}_{\mathrm{i}}, \mathrm{V}_{\mathrm{j}}\right)= & \left\{\left(\mathrm{n}_{\mathrm{i}}, \mathrm{n}_{\mathrm{j}}, \mathrm{b}_{0}\left(\neg \mathrm{ov}\left(\operatorname{mes}\left(\mathrm{n}_{\mathrm{i}}\right), \operatorname{mes}\left(\mathrm{n}_{\mathrm{j}}\right)\right)\right)\right) \mid\right. \\
& \left.\left(\mathrm{n}_{\mathrm{i}} \in \mathrm{V}_{\mathrm{i}}\right) \text { and }\left(\mathrm{n}_{\mathrm{j}} \in \mathrm{V}_{\mathrm{j}}\right)\right\}
\end{aligned}
$$

An edge $\left(\mathrm{n}_{\mathrm{i}}, \mathrm{n}_{\mathrm{j}}, \mathrm{b}_{0}\left(\neg \mathrm{ov}\left(\operatorname{mes}\left(\mathrm{n}_{\mathrm{i}}\right)\right.\right.\right.$, mes $\left.\left.\left.\left(\mathrm{n}_{\mathrm{j}}\right)\right)\right)\right)$ designates the assumption that the messages represented by the nodes $\mathrm{n}_{\mathrm{i}}$ and $\mathrm{n}_{\mathrm{j}}$ (these are mes $\left(n_{i}\right)$ and mes $\left(n_{j}\right)$ ) overlap with each other and is weighted by a degree of belief in the falsity of this assumption $\mathrm{b}_{0}\left(\neg \mathrm{ov}\left(\operatorname{mes}\left(\mathrm{n}_{\mathrm{i}}\right), \operatorname{mes}\left(\mathrm{n}_{\mathrm{j}}\right)\right)\right)$. The overlap relation is denoted by the predicate ov $\left(\operatorname{mes}\left(n_{i}\right)\right.$,mes $\left.\left.\left(n_{j}\right)\right)\right)$.

The degrees of belief $b_{0}$ are computed according to the formula:

$$
\begin{aligned}
& \mathrm{b}_{0}\left(\neg \mathrm{Ov}\left(\mathrm{m}_{\mathrm{i}}, \mathrm{m}_{\mathrm{j}}\right)\right)= \\
& \sum_{\mathrm{U} \subseteq\{1, \ldots, 6\}}(-1)^{|\mathrm{U}|+1}\left\{\prod_{\mathrm{u} \in \mathrm{U}} \mathrm{b}_{\mathrm{u}}\left(\neg \mathrm{OV}\left(\mathrm{m}_{\mathrm{i}}, \mathrm{m}_{\mathrm{j}}\right)\right)\right\}(\mathrm{I})
\end{aligned}
$$

The functions $b_{1}, \ldots, b_{6}$ used in (I) are defined in Section 4.2 below. After computing the beliefs $b_{0}$ for all the edges of $\operatorname{IOG}\left(\mathrm{I}_{\mathrm{i}}, \mathrm{I}_{\mathrm{j}}\right)$, the overlaps between the messages in $I_{i}$ and $I_{j}$ are detected in a two-step process. First, the most likely candidate overlaps are identified by selecting a subset $\mathrm{O}\left(\mathrm{V}_{\mathrm{i}}, \mathrm{V}_{\mathrm{j}}\right)$ of $\mathrm{E}\left(\mathrm{V}_{\mathrm{i}}, \mathrm{V}_{\mathrm{j}}\right)$ which is a total morphism between $\mathrm{V}_{\mathrm{i}}$ and $\mathrm{V}_{\mathrm{j}}$ and minimises the function:

$$
\sum \quad \mathrm{b}_{0}\left(\neg \mathrm{ov}\left(\operatorname{mes}\left(\mathrm{n}_{\mathrm{u}}\right), \operatorname{mes}\left(\mathrm{n}_{\mathrm{w}}\right)\right)\right)
$$

$\left(\mathrm{nu}, \mathrm{n}_{\mathrm{w}}, \mathrm{b} 0\left(\neg \mathrm{ov}\left(\operatorname{mes}(\mathrm{nu}), \operatorname{mes}\left(\mathrm{n}_{\mathrm{w}}\right)\right)\right)\right) \in \mathrm{O}\left(\mathrm{Vi}_{\mathrm{i}} \mathrm{Vj}\right)$

The morphism $\mathrm{O}\left(\mathrm{V}_{\mathrm{i}}, \mathrm{V}_{\mathrm{j}}\right)$ is selected using an algorithm known as the Hungarian method [10]. In the second step, $\mathrm{O}\left(\mathrm{V}_{\mathrm{i}}, \mathrm{V}_{\mathrm{j}}\right)$ is further restricted to include only the edges $\left(\mathrm{n}_{\mathrm{u}}, \mathrm{n}_{\mathrm{w}}, \mathrm{b}_{0}(\neg \mathrm{ov}\right.$ $\left.\left(\operatorname{mes}\left(\mathrm{n}_{\mathrm{u}}\right), \operatorname{mes}\left(\mathrm{n}_{\mathrm{w}}\right)\right)\right)$ whose non overlap belief does not exceed a particular threshold value $b_{t}$, that is:

$$
\mathrm{b}_{0}\left(\neg \mathrm{ov}\left(\operatorname{mes}\left(\mathrm{n}_{\mathrm{u}}\right), \operatorname{mes}\left(\mathrm{n}_{\mathrm{v}}\right)\right)\right) \leq \mathrm{b}_{\mathrm{t}}
$$

\subsection{Underlying belief functions}

The functions $b_{1}, \ldots, b_{6}$ used in (I) are computed according to six indicators of the non existence of overlap relations between messages, namely the indicator of non-equivalent message operations, and the indicators of different message senders, receivers, stereotypes, activators and activations. These functions are defined in the following.

\subsubsection{Belief due to non-equivalent operations: $\mathbf{b}_{1}$}

$b_{1}$ is the belief in the non existence of an overlap between two messages $m_{i}$ and $m_{j}$ computed due the non equivalent operations indicator. According to this indicator, two messages do not overlap if they invoke operations which do not override the same most general operation in a class model. $b_{1}$ is defined as follows:

Definition 1: The functional form of $\mathrm{b}_{1}$ is:

$$
\begin{aligned}
& \mathrm{b}_{1}\left(\neg \mathrm{ov}\left(\mathrm{m}_{\mathrm{i}}, \mathrm{m}_{\mathrm{j}}\right)\right)=\alpha_{1} \times \mathrm{d}_{1}\left(\mathrm{~m}_{\mathrm{i}}, \mathrm{m}_{\mathrm{j}}\right) \\
& \mathrm{b}_{1}\left(\mathrm{ov}\left(\mathrm{m}_{\mathrm{i}}, \mathrm{m}_{\mathrm{j}}\right)\right)=0
\end{aligned}
$$

where

- $\mathrm{d}_{1}\left(\mathrm{~m}_{\mathrm{i}}, \mathrm{m}_{\mathrm{j}}\right)=\left(\left|\mathrm{Ops}\left(\mathrm{o}_{\mathrm{i}}\right)-\operatorname{Ops}\left(\mathrm{o}_{\mathrm{j}}\right)\right|+\mid \mathrm{Ops}\left(\mathrm{o}_{\mathrm{j}}\right)-\right.$ $\left.\operatorname{Ops}\left(\mathrm{o}_{\mathrm{i}}\right) \mid\right) /\left|\operatorname{Ops}\left(\mathrm{o}_{\mathrm{i}}\right) \cup \operatorname{Ops}\left(\mathrm{o}_{\mathrm{j}}\right)\right|$

- $o_{i}$ is the operation invoked by $m_{i}$ and $o_{j}$ is the operation invoked by $\mathrm{m}_{\mathrm{j}}$

- Ops $\left(\mathrm{o}_{\mathrm{i}}\right)$ is the set of operations having the same signature with $\mathrm{O}_{\mathrm{i}}$ which are defined in the superclasses of the class that defines $\mathrm{o}_{\mathrm{i}}$ and which do not override any operation with the same signature as $\mathrm{o}_{\mathrm{i}}$ 
- $\alpha_{1}$ is the expected ratio of messages $m_{i}$ and $m_{j}$ with non-equivalent operations which do not overlap $\left(0 \leq \alpha_{1} \leq 1\right)$

In single inheritance class models, Ops $\left(\mathrm{o}_{\mathrm{i}}\right)$ and $\operatorname{Ops}\left(\mathrm{o}_{\mathrm{j}}\right)$ are always singleton sets and, therefore, $\mathrm{b}_{1}$ is always equal to $\alpha_{1}$ or 0 . In multiple inheritance class models, where there may be ambiguities regarding the most general operation that is overridden by an operation, each of $\mathrm{Ops}\left(\mathrm{o}_{\mathrm{i}}\right)$ and $\operatorname{Ops}\left(\mathrm{o}_{\mathrm{j}}\right)$ may have more than one elements (see for example ). In such cases, $b_{1}$ can take any value in the range $\left[0, \ldots, \alpha_{1}\right]$.

\subsubsection{Belief due to different message senders: $b_{2}$}

$b_{2}$ is the belief in the non existence of an overlap between two messages $m_{i}$ and $m_{j}$ computed due to the indicator of different senders. According to this indicator, two messages do not overlap if they are sent by objects which are instances of different classes (called "senders" in UML). $\mathrm{b}_{2}$ is defined as follows:

Definition 2: The functional form of $\mathrm{b}_{2}$ is:

$$
\begin{aligned}
& \mathrm{b}_{2}\left(\neg \mathrm{ov}\left(\mathrm{m}_{\mathrm{i}}, \mathrm{m}_{\mathrm{j}}\right)\right)=\alpha_{2} \times \mathrm{d}_{2}\left(\mathrm{~s}_{\mathrm{i}}, \mathrm{s}_{\mathrm{j}}\right) \\
& \mathrm{b}_{2}\left(\mathrm{ov}\left(\mathrm{m}_{\mathrm{i}}, \mathrm{m}_{\mathrm{j}}\right)\right)=0
\end{aligned}
$$

where

- $s_{i}$ is the class of the object that sends $m_{i}$ and $s_{j}$ is the class of the object that sends $\mathrm{m}_{\mathrm{j}}$

- $\mathrm{d}_{2}\left(\mathrm{c}_{\mathrm{i}}, \mathrm{c}_{\mathrm{j}}\right)=\sum_{\mathrm{x} \in \mathrm{NCSij}} \operatorname{SD}(\mathrm{x})^{-1} / \sum_{\mathrm{y} \in \operatorname{ASSij}} \operatorname{SD}(\mathrm{y})^{-1}$ if $c_{i}$ and $c_{i}$ are specified in the models

- $\mathrm{d}_{2}\left(\mathrm{c}_{\mathrm{i}}, \mathrm{c}_{\mathrm{j}}\right)=1$ if $\mathrm{c}_{\mathrm{i}}$ or $\mathrm{c}_{\mathrm{i}}$ is not specified in the models

- $\mathrm{NCS}_{\mathrm{ij}}$ is the set difference of the superclasses of $\mathrm{c}_{\mathrm{i}}$ and $c_{j}$

- $\mathrm{SD}(\mathrm{x})$ is the maximum length (number of links) of the paths connecting a class $\mathrm{x}$ with the most general class of its generalisation hierarchy, called specialisation depth of $\mathrm{x}$

- $\alpha_{2}$ is the expected ratio of messages $m_{i}$ and $m_{j}$ with different senders which do not overlap $(0 \leq$ $\left.\alpha_{2} \leq 1\right)$

$b_{2}$ measures the belief in the non-identity of two classes based on the number of their non common superclasses and the relative depth of them in the generalisation graph(s) of the models.

\subsubsection{Belief due to different message receivers: $b_{3}$}

$b_{3}$ is the belief in the non existence of an overlap between two messages $\mathrm{m}_{\mathrm{i}}$ and $\mathrm{m}_{\mathrm{j}}$ computed due to the indicator of different receivers. According to this indicator, two messages do not overlap if they are received by objects which are instances of different classes (called "receivers" in UML). Similarly to $b_{2}, b_{3}$ is defined as follows:

Definition 3: The functional form of $\mathrm{b}_{3}$ is:

$$
\begin{aligned}
& \mathrm{b}_{3}\left(\neg \mathrm{ov}\left(\mathrm{m}_{\mathrm{i}}, \mathrm{m}_{\mathrm{j}}\right)\right)=\alpha_{3} \times \mathrm{d}_{2}\left(\mathrm{r}_{\mathrm{i}}, \mathrm{r}_{\mathrm{j}}\right) \\
& \mathrm{b}_{3}\left(\mathrm{ov}\left(\mathrm{m}_{\mathrm{i}}, \mathrm{m}_{\mathrm{j}}\right)\right)=0
\end{aligned}
$$

where

- $r_{i}$ is the class of the object that receives $m_{i}$ and $r_{j}$ is the class of the object that receives $\mathrm{m}_{\mathrm{j}}$

- $\mathrm{d}_{2}$ is as defined in Definition 3 above

- $\alpha_{3}$ is the expected ratio of messages $m_{i}$ and $m_{j}$ with different receivers which do not overlap $(0 \leq$ $\left.\alpha_{3} \leq 1\right)$

\subsubsection{Belief due to different message stereotypes: $\mathbf{b}_{4}$}

$\mathrm{b}_{4}$ is the belief in the non existence of an overlap between two messages $\mathrm{m}_{\mathrm{i}}$ and $\mathrm{m}_{\mathrm{j}}$ computed due to the indicator of different stereotypes. According to this indicator, two messages do not overlap if they are have different stereotypes and, therefore, they belong to semantically different groups of messages. $b_{4}$ is defined as follows:

Definition 4: The functional form of $\mathrm{b}_{4}$ is:

$$
\begin{aligned}
& \mathrm{b}_{4}\left(\neg \mathrm{Ov}\left(\mathrm{m}_{\mathrm{i}}, \mathrm{m}_{\mathrm{j}}\right)\right)=\alpha_{4} \times \mathrm{d}_{4}\left(\mathrm{~m}_{\mathrm{i}}, \mathrm{m}_{\mathrm{j}}\right) \\
& \mathrm{b}_{4}\left(\neg \mathrm{Ov}\left(\mathrm{m}_{\mathrm{i}}, \mathrm{m}_{\mathrm{j}}\right)\right)=0
\end{aligned}
$$

where

- $\mathrm{d}_{4}\left(\mathrm{~m}_{\mathrm{i}}, \mathrm{m}_{\mathrm{j}}\right)=\left(\left|\operatorname{Stypes}\left(\mathrm{m}_{\mathrm{i}}\right) \quad-\operatorname{Stypes}\left(\mathrm{m}_{\mathrm{j}}\right)\right|+\right.$ $\left.\left|\operatorname{Stypes}\left(\mathrm{m}_{\mathrm{j}}\right)-\operatorname{Stypes}\left(\mathrm{m}_{\mathrm{i}}\right)\right|\right) \quad / \quad \mid \operatorname{Stypes}\left(\mathrm{m}_{\mathrm{i}}\right) \cup$ $\operatorname{Stypes}\left(\mathrm{m}_{\mathrm{j}}\right)$

- $\operatorname{Stypes}\left(\mathrm{m}_{\mathrm{i}}\right)$ and $\operatorname{Stypes}\left(\mathrm{m}_{\mathrm{j}}\right)$ are the sets of the stereotypes of the messages $\mathrm{m}_{\mathrm{i}}$ and $\mathrm{m}_{\mathrm{j}}$, respectively

- $\alpha_{4}$ is the expected ratio of messages $m_{i}$ and $m_{j}$ with different stereotypes which do not overlap $\left(0 \leq \alpha_{4} \leq 1\right)$

$b_{4}$ measures a belief based on the number of the non common stereotypes of two messages.

\subsubsection{Belief due to different message activators: $\mathbf{b}_{5}$}

$b_{5}$ is the belief in the non existence of an overlap between two messages $\mathrm{m}_{\mathrm{i}}$ and $\mathrm{m}_{\mathrm{j}}$ computed due to the indicator of different activators. According to this indicator, two messages do not overlap if they are dispatched by non overlapping messages (called "activators" in UML) or equivalently (in terms of our definition of message overlaps) in the course of execution of operations with different implementations. $b_{5}$ is defined as follows: 
Definition 5: The functional form of $\mathrm{b}_{5}$ is:

$$
\begin{aligned}
\mathrm{b}_{5}\left(\neg \mathrm{OV}\left(\mathrm{m}_{\mathrm{i}}, \mathrm{m}_{\mathrm{j}}\right)\right)= & \alpha_{4} \times \Sigma_{\mathrm{U} \subseteq\{1, \ldots, 4\}}(-1)^{|\mathrm{U}|+1}\left\{\Pi_{\mathrm{u} \in \mathrm{U}}\right. \\
& \left.\mathrm{b}_{\mathrm{u}}\left(\neg \mathrm{OV}\left(\mathrm{m}_{\mathrm{i}}, \mathrm{m}_{\mathrm{j}}\right)\right)\right\} \\
\mathrm{b}_{5}\left(\mathrm{ov}\left(\mathrm{m}_{\mathrm{i}}, \mathrm{m}_{\mathrm{j}}\right)\right)= & 0
\end{aligned}
$$

where

- $\mathrm{b}_{\mathrm{u}}(\mathrm{u}=1, \ldots, 4)$ are as defined in Definitions 1-4

- $\alpha_{5}$ is the expected ratio of messages $\mathrm{m}_{\mathrm{i}}$ and $\mathrm{m}_{\mathrm{j}}$ with different activators which do not overlap $(0$ $\left.\leq \alpha_{5} \leq 1\right)$

$b_{5}$ is an approximation of $b_{6}$ used to avoid a double full analysis of activators in detecting overlaps.

\subsubsection{Belief due to different message activations: $b_{6}$}

$b_{6}$ is the belief in the non existence of an overlap between two messages $m_{i}$ and $m_{j}$ computed due to the indicator of different activations. According to this indicator, two messages do not overlap if they dispatch non overlapping messages (called "activations" in UML) or, equivalently (in terms of our definition of overlap), they invoke operations with different implementations. $b_{6}$ is defined as follows:

Definition 6: The functional form of $\mathrm{b}_{6}$ is:

where

$$
\mathrm{b}_{6}\left(\neg \mathrm{ov}\left(\mathrm{m}_{\mathrm{i}}, \mathrm{m}_{\mathrm{j}}\right)\right)=\alpha_{6} \times \mathrm{d}_{6}\left(\mathrm{~m}_{\mathrm{i}}, \mathrm{m}_{\mathrm{j}}\right)
$$$$
\mathrm{b}_{6}\left(\mathrm{ov}\left(\mathrm{m}_{\mathrm{i}}, \mathrm{m}_{\mathrm{j}}\right)\right)=0
$$

$$
\text { - } \begin{aligned}
\mathrm{d}_{6}\left(\mathrm{~m}_{\mathrm{i}}, \mathrm{m}_{\mathrm{j}}\right)= & \left(\operatorname { m i n } _ { \mathrm { X } \in \operatorname { M o r p h i s m s } ( \mathrm { i } , \mathrm { j } ) } \left(\sum_{(\mathrm{mu}, \mathrm{mv}) \in \mathrm{X}}\right.\right. \\
& \mathrm{b}_{\mathrm{o}}\left(\neg \mathrm{OV}\left(\mathrm{m}_{\mathrm{u}}, \mathrm{m}_{\mathrm{v}}\right)\right)+\max \left(\left|\mathrm{A}_{\mathrm{i}}\right|-\left|\mathrm{A}_{\mathrm{j}}\right|,\left|\mathrm{A}_{\mathrm{j}}\right|\right. \\
& \left.\left.-\left|\mathrm{A}_{\mathrm{i}}\right|\right)\right) / \max \left(\left|\mathrm{A}_{\mathrm{i}}\right|,\left|\mathrm{A}_{\mathrm{j}}\right|\right)
\end{aligned}
$$$$
\text { if } A_{i} \neq \varnothing \text { and } A_{j} \neq \varnothing
$$

$$
\mathrm{d}_{6}\left(\mathrm{~m}_{\mathrm{i}}, \mathrm{m}_{\mathrm{j}}\right)=1 \text { if } \mathrm{A}_{\mathrm{i}}=\varnothing \text { or } \mathrm{A}_{\mathrm{j}}=\varnothing \text {. }
$$

- $\mathrm{A}_{\mathrm{i}}$ and $\mathrm{A}_{\mathrm{j}}$ are the sets of the messages which are dispatched by the messages $\mathrm{m}_{\mathrm{i}}$ and $\mathrm{m}_{\mathrm{j}}$, respectively.

- $M(i, j)$ is the set of all the total morphisms from the messages in $A_{i}$ to the messages in $A_{j}$ if $\left|A_{i}\right| \leq$ $\left|A_{j}\right|$ or the set of all the onto morphisms from the messages in $A_{i}$ to the messages in $A_{j}$ if $\left|A_{j}\right|<\left|A_{i}\right|$.

- $\mathrm{b}_{\mathrm{o}}\left(\neg \mathrm{Ov}\left(\mathrm{m}_{\mathrm{u}}, \mathrm{m}_{\mathrm{v}}\right)\right)$ is as defined by formula (I).

- $\alpha_{6}$ is the expected ratio of messages $m_{i}$ and $m_{j}$ with different activators which do not overlap ( 0 $\left.\leq \alpha_{6} \leq 1\right)$

$b_{6}$ measures a belief based on a recursive check for overlaps between the messages which are dispatched by the messages of concern.

\subsubsection{Properties of used belief functions}

As we prove in [21], the functions $b_{1}, \ldots, b_{6}$ are all distance metrics. They also satisfy the axioms that define Dempster-Shafer basic probability assignments [19] and, as a consequence, they are legitimately interpreted as probability assignments of this kind. The main reason for this interpretation is that $b_{1}, \ldots, b_{6}$ have been defined so as to assign measures of belief in the non existence of an overlap relation between messages and not to the existence of such relations, that is $b_{i}\left(o v\left(m_{u}, m_{v}\right)\right)=$ 0 for all $i=1, \ldots, 6$. This is because of the indicators underlying these functions. More specifically, it has to be appreciated that while the presence of these indicators in the case of two messages does indicate that an overlap relation between them is unlikely, the absence of the indicators does not provide evidence that such an overlap relation exists between the messages.

Consider, for instance, the indicator of different receivers. When two messages have different receivers in interaction diagrams, it is more plausible to assume that they invoke operations with different implementations than to assume that they invoke operations with the same implementation. However, when two messages have the same receiver we cannot conclude that an overlap relation between them exists. Similar arguments apply for the other indicators.

The functions $b_{i}(i=1, \ldots, 6)$ have been defined so as to: $b_{i}\left(o v\left(m_{u}, m_{v}\right)\right)+b_{i}\left(\neg o v\left(m_{u}, m_{v}\right)\right) \leq 1$. Belief functions that have this property of assigning a total belief of possibly less than 1 to a proposition and its negation are valid as Dempster-Shafer basic probability assignments but not as classical probability functions for which it must always be that $\operatorname{Prob}(E)+\operatorname{Prob}(\neg E)=1[16]$.

As we prove in [21], the functional form of $b_{0}$ is derived from the combination of the belief functions $b_{1}, \ldots, b_{6}$ using the rule of the orthogonal sum of the Dempster-Shafer theory and measures the belief jointly committed to $\neg \mathrm{ov}\left(\mathrm{m}_{\mathrm{i}}, \mathrm{m}_{\mathrm{j}}\right)$ by these functions. $b_{0}$ has also been proved to be a distance metric (see [21]). These properties of $b_{0}$ guarantee the following intuitively plausible relations between its outputs:

- $\mathrm{b}_{0}\left(\neg \mathrm{ov}\left(\mathrm{m}_{\mathrm{i}}, \mathrm{m}_{\mathrm{k}}\right)\right) \leq \mathrm{b}_{0}\left(\neg \mathrm{ov}\left(\mathrm{m}_{\mathrm{i}}, \mathrm{m}_{\mathrm{j}}\right)\right)+\mathrm{b}_{0}(\neg \mathrm{ov}$ $\left(\mathrm{m}_{\mathrm{j}}, \mathrm{m}_{\mathrm{k}}\right)$ ) (due to triangularity of distance metrics)

- $\mathrm{b}_{0}\left(\neg \mathrm{ov}\left(\mathrm{m}_{\mathrm{i}}, \mathrm{m}_{\mathrm{j}}\right)\right)=\mathrm{b}_{0}\left(\neg \mathrm{ov}\left(\mathrm{m}_{\mathrm{j}}, \mathrm{m}_{\mathrm{i}}\right)\right)$ (due to symmetry of distance metrics)

- $\mathrm{b}_{0}\left(\neg \mathrm{ov}\left(\mathrm{m}_{\mathrm{i}}, \mathrm{m}_{\mathrm{j}}\right) \wedge \mathrm{ov}\left(\mathrm{m}_{\mathrm{j}}, \mathrm{m}_{\mathrm{i}}\right)\right)=0$ (due to axiomatic foundation of Dempster-Shafer basic probability assignments) 
Draft - An updated version of this paper has appeared in the Proceedings of the $7^{\text {th }}$ International Conference on Object Oriented Information Systems

Finally, the measure that results from the minimisation of formula (II) is an upper bound of:

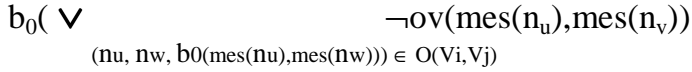

This is the belief in the proposition that at least one of the overlap relations in $\mathrm{O}\left(\mathrm{V}_{\mathrm{i}}, \mathrm{V}_{\mathrm{j}}\right)$ is wrong.

\subsection{Example of detection of optimal overlap morphism}

As an example of detecting overlaps consider the sequence diagrams $I_{1}$ and $I_{2}$ in Figures 4 and 5 , respectively. These diagrams specify interactions between the objects in a library system which occur when a user uses it to search for a library item by keywords referring to the title $\left(\mathrm{I}_{1}\right)$ or to the author of an item $\left(\mathrm{I}_{2}\right)$.

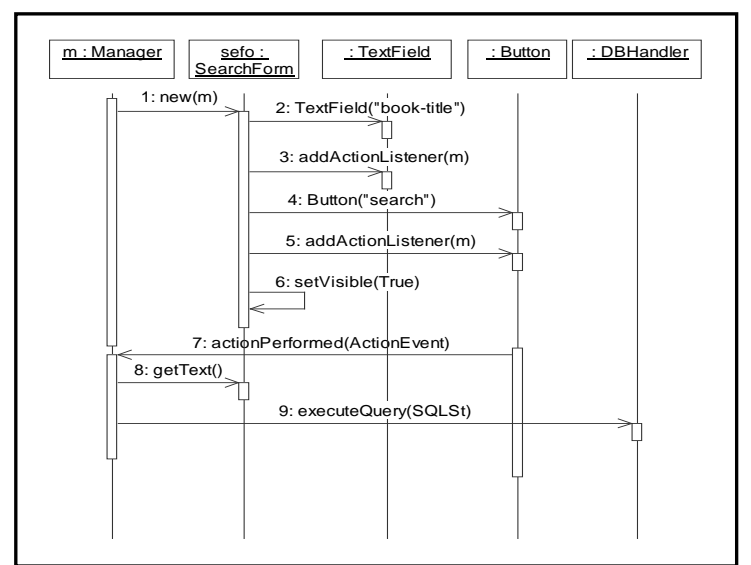

Figure 4: Interaction diagram $\mathrm{I}_{1}$ - SearchByTitle

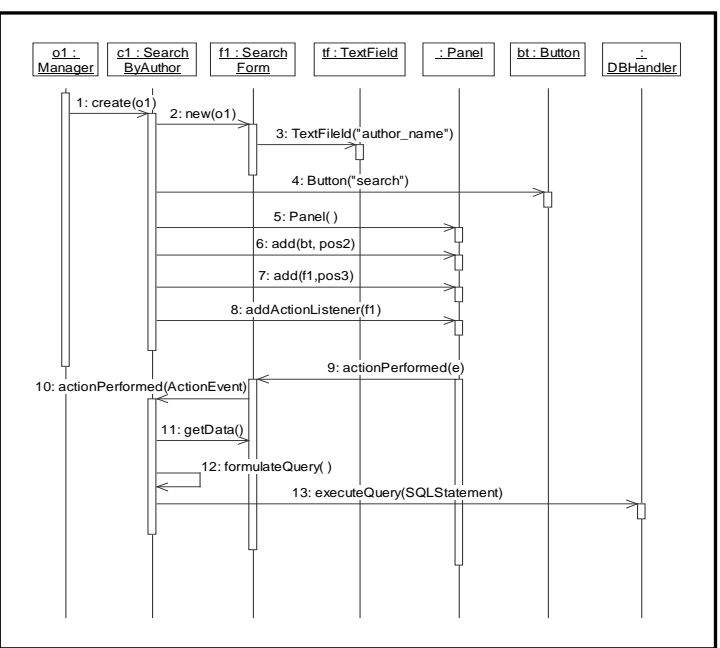

Figure 5: Interaction diagram $\mathrm{I}_{2}$ - SearchByAuthor

$\mathrm{I}_{1}$ and $\mathrm{I}_{2}$ assume the event-driven GUI implementation model of the Java 1.1 Abstract Window Toolkit [20]. More specifically, in $\mathrm{I}_{1}$ the class SearchForm has been declared as a subclass of the Java AWT component Panel (see Figure 6) and has a text field to let the user to type in the keywords that should be searched within the titles of items. In $\mathrm{I}_{2}$, the class SearchByAuthor incorporates a panel composed of a text field and a button that enable the user type in the author keywords and initiate the search.

When a search is initiated the system gets the search keywords from the appropriate UI component (see messages 8:getText() in $\mathrm{I}_{1}$ and 11:getData() in $\mathrm{I}_{2}$ ). formulates a query (see message 12:formulateQuery() in $\mathrm{I}_{2}$ ), and forwards this query to a database driver to execute it (see messages 9:executeQuery(SQLSt) in $\mathrm{I}_{1}$ and 13:executeQuery(SQLStatement) in $\mathrm{I}_{2}$ ).

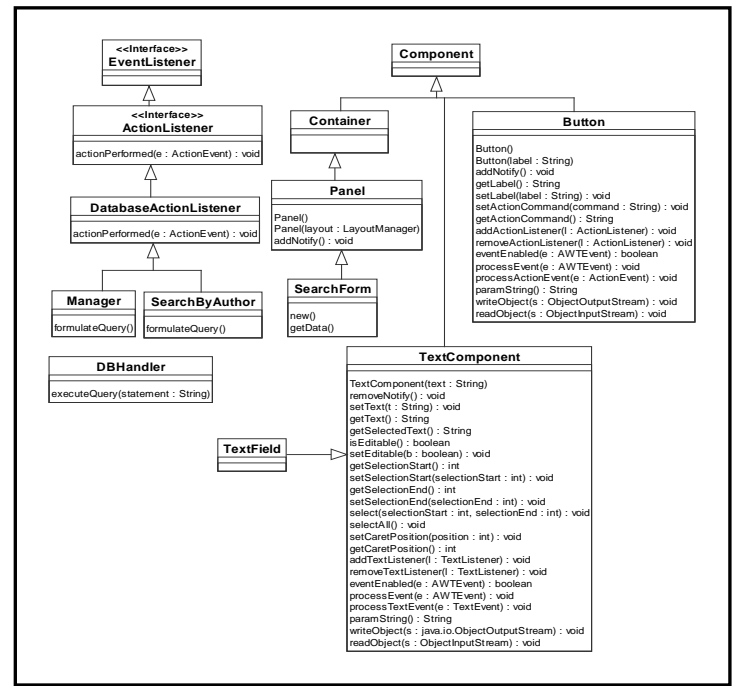

Figure 6: Generalisation graph of the classes in $\mathrm{I}_{1}$ and $\mathrm{I}_{2}$

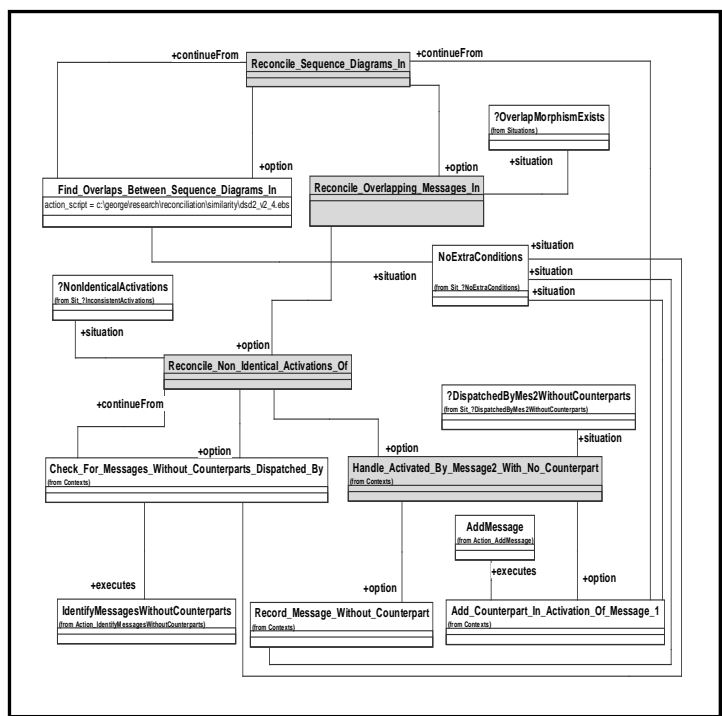

Figure 7: Part of the Reconciliation+ process model 
The detection of overlaps can be activated during the enactment of the Reconciliation+ process model by selecting the executable context Find_Overlaps_Between_Sequence_Diagrams_In shown in Figure 7. This figure shows part of the Reconciliation+ process model (the choice contexts are grey and the executable contexts are white).

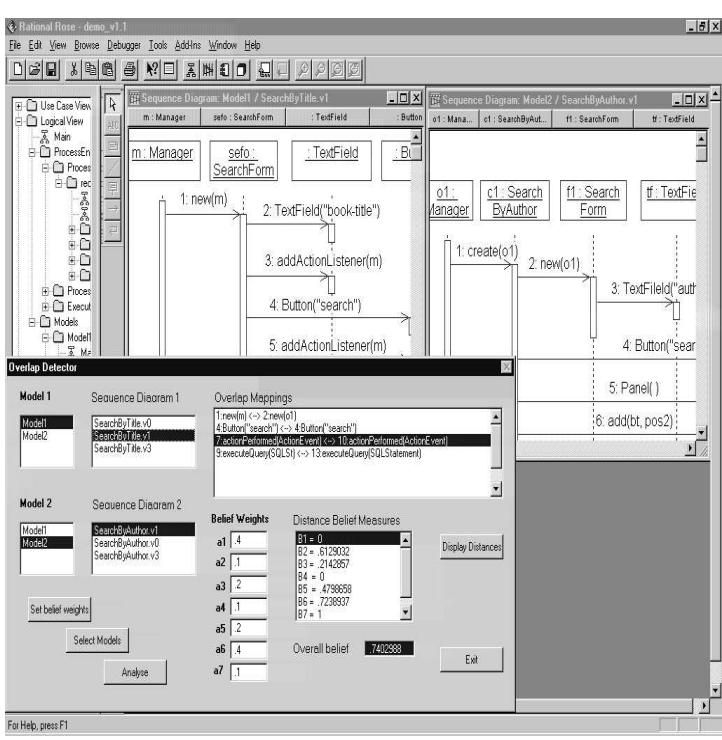

Figure 8: Screenshot of Overlap Detector

Find_Overlaps_Between_Sequence_Diagrams_In is instantiated for a default package of the underlying tool repository that includes the models to be reconciled and is an external action executable context that invokes the overlap detector.

As shown in Figure 8, the overlaps detected between the messages of $\mathrm{I}_{1}$ and $\mathrm{I}_{2}$ are:

- ov(1:new $(m), 2: n e w(o 1))-b_{1}=0, b_{2}=0.21, b_{3}$ $=0, b_{4}=0, b_{5}=1, b_{6}=0.93$, and $b_{0}=0.559$

- ov(4:Button("search"), 4:Button("search")) - $\mathrm{b}_{1}$ $=0, b_{2}=1, b_{3}=0, b_{4}=0, b_{5}=0.52, b_{6}=1$, and $\mathrm{b}_{0}=0.564$

- ov(7:actionPerformed(ActionEvent),

10: actionPerformed(ActionEvent)) - $\mathrm{b}_{1}=0, \mathrm{~b}_{2}$ $=0.61, b_{3}=0.21, b_{4}=0, b_{5}=0.48, b_{6}=0.72$, and $\mathrm{b}_{0}=0.48$

- ov(9:executeQuery(SQLSt),

13: executeQuery $(S Q L$ Statement $))-\mathrm{b}_{1}=0, \mathrm{~b}_{2}=$ $0.21, \mathrm{~b}_{3}=0, \mathrm{~b}_{4}=0, \mathrm{~b}_{5}=0.1, \mathrm{~b}_{6}=1$, and $\mathrm{b}_{0}=$ 0.48

The above relations were detected using a threshold value of 0.6 for $b_{t}$ and the $\alpha_{i}$ values shown in Figure 8.
The overlaps detected by the Overlap Detector are represented in the process enactment trace as shown in Figure 9. Each of the detected overlap relations is represented by an object which is associated with the messages involved in the relation. This object also stores the measures generated by the functions $b_{1}, \ldots, b_{6}$ as values of specific attributes of its own.

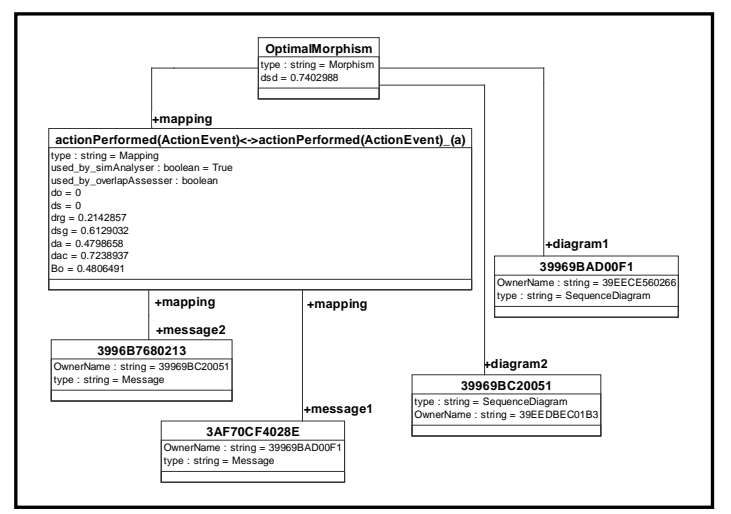

Figure 9: Optimal morphism in the process execution trace

For example, the object "actionPerformed(ActionEvent)<-> actionPerformed(ActionEvent)" represents the overlap relation between the so called messages of $I_{1}$ and $I_{2}$, and is associated with objects that represent these two messages (they appear with their internal identifiers in Figure 8). It also stores their $d_{3}$ distance (as the value of its attribute $d r g$ ) and is associated with an object that represents the selected optimal morphism between $I_{1}$ and $I_{2}$ (called "OptimalMorphism"). All these objects are stored in a special package of the tool repository called "ExecutionClasses" which is used to store information generated during the enactment of the process model of the method.

\section{Specification of consistency rules and detection of inconsistencies}

In our method, consistency rules are specified as internal actions of a special kind of executable internal action contexts, called "consistency check contexts". These contexts are instances of the metaclass ConsistencyCheckContext in Figure 2.

As we discussed in Section 3.1, an internal action can generally be specified as a sequence of set, save, or modification operations. In the case of consistency check contexts, however, the internal actions are restricted not to include any modification operations. This restriction guarantees that the execution of a consistency check will not modify the contents of the underlying models. 
Furthermore, the last operation in the sequence of operations that specify a consistency rule must be a SaveOperation. Save operations save a set in the process execution trace (i.e. the special package called "ExecutionClasses"). In the case of an action that specifies a consistency rule, this set includes the model elements (messages) which breach the rule. This restriction realises a well-formedness rule for the Reconciliation+ process models which guarantees that the results of a consistency check will always be made temporarily available to the enactment of the process ${ }^{2}$.

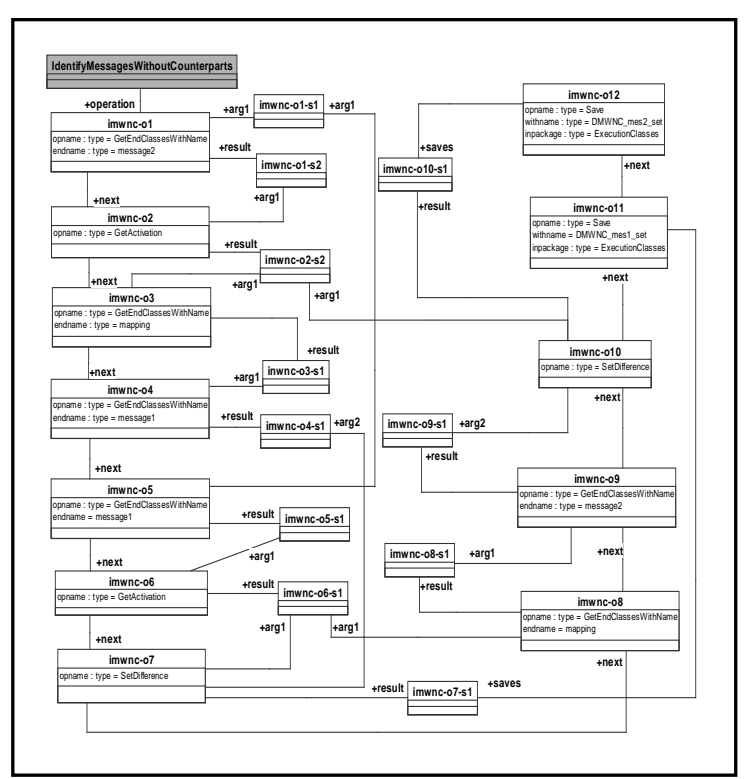

Figure 10: Specification of action IdentifyMessagesWithoutCounterparts

Next, we give an example of a consistency rule specified according to the scheme outlined above and show when exactly it may be checked in the overall process of Reconciliation+ and the results of its checking.

\subsection{Specification of consistency rules: an example}

As an example of a consistency rule specified according to the scheme outlined above, consider again the rule CR1. As discussed in Section 1, CR1 requires that for each message $\mathrm{x}$ activated by a message $m_{i}$ which overlaps with a message $m_{j}$ there must be a message $y$ activated by $m_{j}$ that overlaps with $\mathrm{x}$ and vice versa.

2 They can also become permanently available through an inconsistency handling action that saves an inconsistency recorded in the process trace in a special package called Inconsistencies whose contents persist the completion of a process enactment session.
In the process model of Reconciliation+, CR1 is specified as the action IdentifyMessagesWithoutCounterparts of the consistency check context Check_For_Messages_Without_Counterparts_Disp atched_By shown in Figure 7. To check this rule against a pair of overlapping messages, the developer can select a decision that applies Check_For_Messages_Without_Counterparts_Disp atched_By to the object that represents the overlap relation detected these messages (see Figure 9). This overlap relation will be referred to as the "selected overlap object" in the following.

As shown in Figure 10, IdentifyMessagesWithoutCounterparts is specified as a sequence of the following operations:

1. imwnc-ol - imwnc-ol is an instance of the unary set operation GetEndClassesWithName which returns in its result set the classes which are attached to the opposite association ends of the classes in its argl set having the same name as the value of its attribute endname. The argl set of imwnc-ol always includes the selected overlap object. Thus, imwnc-ol retrieves the message 2 of the overlap relation represented by it (for example the object "3996B7680213" in Figure 9).

2. imwnc-o2 - imwnc-o2 is an instance of the unary set operation GetActivation. GetActivation returns in its result set the union of the sets of the messages which are activated by the messages in its $\arg 1$ set. Thus, imwnc$o 2$ retrieves the messages which are dispatched by the message 2 of the selected overlap object.

3. imwnc-o3 - imwnc-o3 is an instance of the unary set operation GetEndClassesWithName and retrieves the objects that represent the overlap relations detected for the messages dispatched by message 2 of the selected overlap object.

4. imwnc-o4 - imwnc-o4 is an instance of the unary set operation GetEndClassesWithName and retrieves the messages activated by messagel of the selected overlap object and which overlap with the messages activated by the message 2 of this object.

5. imwnc-o5 - imwnc-o5 is an instance of the unary set operation GetEndClassesWithName and retrieves the messagel of the selected overlap object.

6. imwnc-o6 - imwnc-o6 is an instance of the operation GetActivation and retrieves the set of 
messages which are activated by messagel of the selected overlap object.

7. imwnc-o7 - imwnc-o7 is an instance of the binary set operation SetDifference which returns the set difference between its $\arg 1$ and arg2 set in its result set. Thus, imwnc-o7 returns the messages which have are directly dispatched by the messagel of the selected overlap object and do not overlap with any of the messages which are directly dispatched by the message 2 of this object.

8. imwnc-o8 - Similarly to imwnc-o4, imwnc-o8 retrieves the overlap relations detected for the messages which are directly dispatched by the message1 of the selected overlap object.

9. imwnc-o9 - Similarly to imwnc-o3, imwnc-o9 retrieves the messages which overlap with the messages dispatched by the messagel of the selected overlap object.

10.imwnc-o10 - Similarly to imwnc-o7, imwnc-o10 returns the messages which are directly dispatched by the message 2 of the selected overlap object and which do not overlap with any of the messages dispatched by the messagel of this object.

11.imwnc-ol1 - imwnc-oll is an instance of the SaveOperation which saves with the name indicated by the value of its attribute withname and in the package indicated by the value of its attribute inpackage its saves set. Thus, imwncoll saves in the package ExecutionClasses the set of the messages which are dispatched by messagel of the selected overlap object and do not overlap with any of the messages dispatched by the message 2 of this object. The name of the saved set is DMWNC_mes1_set.

12.imwnc-o12 - Similarly to imwnc-o11, imwnco12 saves in the package ExecutionClasses the set of the messages which are dispatched by message 2 of the selected overlap object and do not overlap with any of the messages which are dispatched by the messagel of this object. The name of the saved set is DMWNC_mes__set.

\subsection{Execution of consistency rules}

A consistency check context becomes available for selection only in certain parts of the reconciliation process (as determined by the process model) and when the situation associated with it is satisfied.

Following the process model of Figure 7, the consistency check context Check_For_Messages_Without_Counterparts_Disp atched_By, for example, may be selected for an overlapping pair of messages only after a designer has selected:

1) the executable context Find_Overlaps_Between_Sequence_Diagrams_ In to detect overlaps relations in the sequence diagrams to be reconciled

2) the choice context Reconcile_Overlapping_Messages_In to start the reconciliation of the overlapping messages detected in these sequence diagrams, and

3) the choice context Reconcile_Non_Identical_Activations_Of to start the reconciliation of the overlapping messages in these sequence diagrams with potentially non identical activations

The selection of Check_For_Messages_Without_Counterparts_Dispatche $d_{-} B y$ for the object representing the overlap between the messages 7:actionPerformed(ActionEvent) in $\mathrm{I}_{1}$ and 10:actionPerformed(ActionEvent)) in $\mathrm{I}_{2}$ generates the following sets of non overlapping messages in the process enactment trace:

- DMWNC_mes1_set $=\{\operatorname{get} T \operatorname{ext}()\}$

- DMWNC_mes2_set = \{getData () , formulateQuery()\}

\section{Handling of Inconsistencies}

Similarly to the consistency checks, the ways of handling inconsistencies are specified as internal actions of a special kind of executable contexts, called inconsistency handling contexts (see Figure 2 ). In this section, we discuss how the situations which determine when these contexts become available and the actions which determine how the inconsistencies can be dealt with are specified.

The process model of Reconciliation+ incorporates contexts for handling inconsistencies which arise as violations of different consistency rules. And in cases where there are more than one possible ways of handling the violations of a particular rule, it includes contexts for each of these ways. These contexts are options of choice contexts in the process model which exist to group the different ways of handling a particular kind of inconsistencies. The situations of these choice contexts are defined in a way that makes them selectable only if there have been recorded violations of the relevant rule in the process enactment trace. 
Draft - An updated version of this paper has appeared in the Proceedings of the $7^{\text {th }}$ International Conference on Object Oriented Information Systems

As an example, consider the inconsistencies recorded as violations of the rule specified by the internal action

of

Check_For_Messages_Without_Counterparts_Dispatche $d \_B y$. As we discussed in Section 5.1, the internal action of this context detects and keeps a record of messages which violate the rule $\mathrm{CR} 1$. The process model defines four contexts for handling these violations, including the contexts:

1) Record_Message_Without_Counterpart, and

2) Add_Counterpart_In_Activation_Of_Message_1.

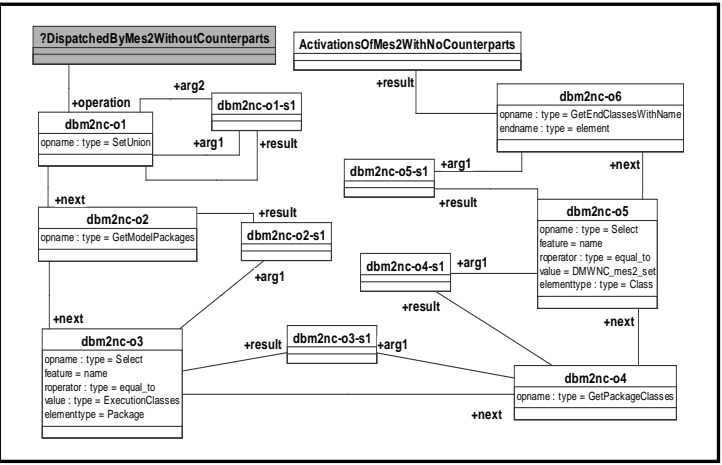

Figure 11: Specification of situation

?DispatchedByMes2WithoutCounterparts

As shown in Figure 7, these contexts are options available from the choice context Handle_Activated_By_Message2_With_No_Counterpart which becomes available only if there are messages that violate $\mathrm{CR} 1$. The satisfiability of this condition is checked when the process enactment engine executes the sequence of the operations of the situation ?DispatchedByMes2WithoutCounterparts of this context shown in Figure 11. As shown in this figure, ?DispatchedByMes2WithoutCounterparts is satisfied only if there is a class called "DMWNC_mes2_set" in the package "ExecutionClasses" of the repository of the Reconciliation+ toolkit representing a non empty set of messages that violate CR1. Note that, as we discussed in Section 5.1, this set is generated by the action of the context Check_For_Messages_Without_Counterparts_Dispatche d_By.

As we discussed in Section 3.4, Handle_Activated_By_Message2_With_No_Counterpart can be applied to any of the messages in the set $D M W N C \_m e s 2$ set. These alternative applications are generated as decisions and are proposed to the developer by the process enactment engine. For example, the options generated from Handle_Activated_By_Message2_With_No_Counterpart in the case of the overlap relation between the messages 7:actionPerformed(ActionPerformed) and 11:actionPerformed(ActionEvent) of the sequence diagrams shown in Figures 4 and 5 are shown in Figure 12. This figure shows a snapshot of the process enactment engine.

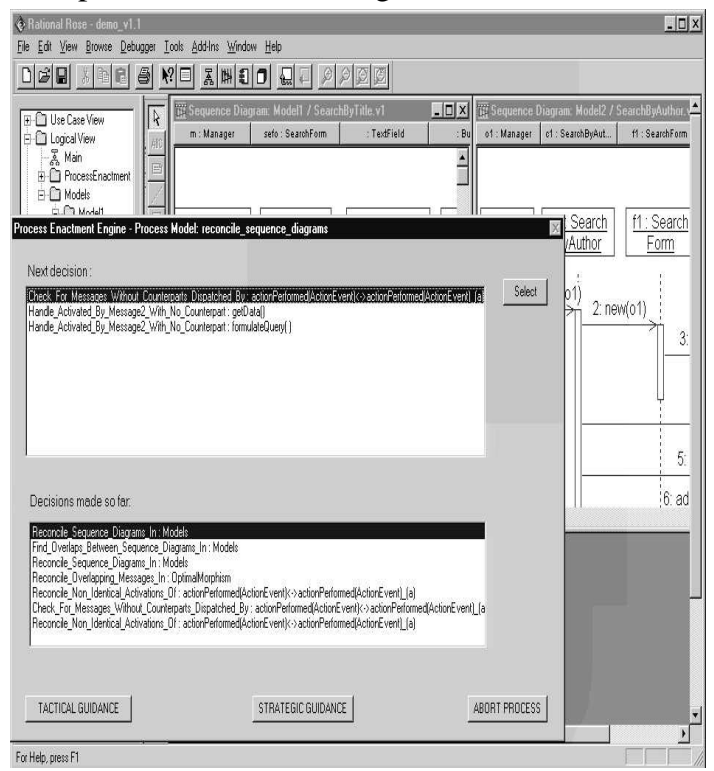

Figure 12: Snapshot of process enactment engine

When one of these decisions is selected, the two alternative contexts for handling this kind of inconsistencies Record_Message_Without_Counterpart and Add_Counterpart_In_Activation_Of_Message_1 also become options available for selection. This is because none of these contexts has any extra conditions in its situation.

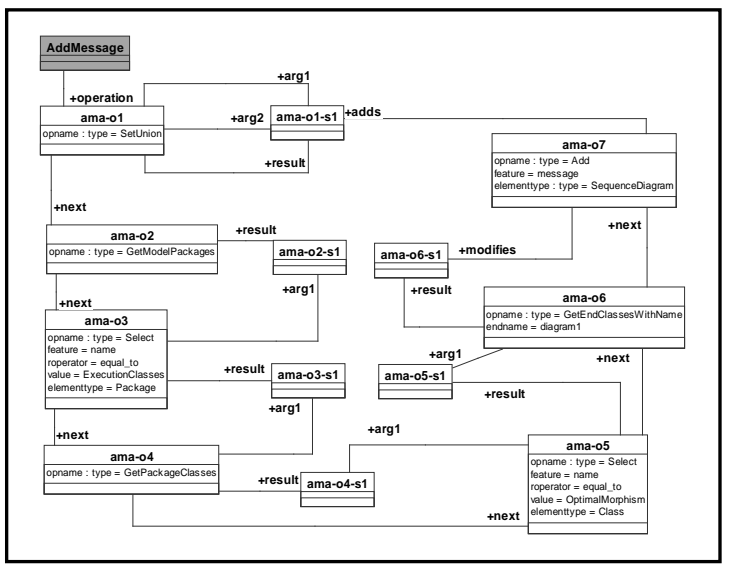

Figure 13: Specification of action AddMessage

Add_Counterpart_In_Activation_Of_Message_l can be selected to create a copy of a message in the activation of a message $m_{j}$ that does not have an overlapping counterpart in the activation of a message $m_{i}$ that overlaps with it and add it to the activation of $\mathrm{m}_{\mathrm{i}}$. 
Record_Message_Without_Counterpart can be activated to make a persistent record (i.e. a record saved after the end of the on-going process enactment trace) of the inconsistency. In the following, we explain the specification of the action of the former context that realises the former way of handling the inconsistency.

The specification of the action AddMessage of Add_Counterpart_In_Activation_Of_Message_l is shown in Figure 13. According to this specification, in executing AddMessage, the process enactment engine first locates the sequence diagram of message $m_{i}$ in the overlap relation $\left(m_{i}, m_{j}\right)$ (see operations ama-o2 to ama-o6), and then adds (see operation ama-o7) to the set of the messages of this diagram a copy of the message without the counterpart (that is the element of the set ama-ols1). ama-o7 is an instance of the operation type AddMessage and is used to add copies of the message in its argl set to each of the sequence diagrams which belong to its modifies set.

\section{Related Work}

A considerable body of research has been concerned with the problem of detecting inconsistencies in software models and documentation. This work has generated techniques for detecting inconsistencies in structured and textbased $[1,3,4,12]$, object-oriented $[2,6,18]$, statebased $[7,8]$, and formal software models $[5,17]$.

Some of the proposed techniques focus on objectoriented models. Glinz [6], for example, has developed a technique that checks behavioural software models expressed as statecharts for deadlocks, reachability and mutual exclusiveness of states. Cheung et al [2] have developed a technique that checks whether the sequence of the execution of operations that is implied by a UML statechart diagram is compliant with the sequence of the executions of operations implied by a UML sequence diagram. Zisman et al [18] have developed a consistency link generator which checks whether UML software models satisfy specific consistency rules. These rules are expressed in XML [2] and the consistency checking is performed using a tool developed using and XML development platform.

A critical survey of all the above techniques may be found in [15].

\section{Conclusions and Further Work}

In this paper, we presented Reconciliation+, a tool supported method developed to guide developers in reconciling models of the behaviour of software systems expressed as UML object interaction diagrams. The method provides a mechanism for detecting overlaps between messages in interaction models, a scheme for specifying consistency rules that overlapping messages must satisfy, and a scheme for specifying ways of handling violations of these rules. The rules and the ways of handling inconsistencies are specified as parts of a process model that is enacted by the method to drive the reconciliation of interaction diagrams. The method is extensible and configurable. This is because developers may add both consistency check and inconsistency handling contexts to its process model. The can also alter the situations of existing contexts.

Currently, we are evaluating the prototype developed for the method in an industrial case study. We are also studying properties of the schemes for expressing the consistency rules and the inconsistency handling contexts in the method. Future work will focus on expanding the built-in process models of the method to enable the capture of rationale for the selection of specific inconsistency handling actions.

\section{Acknowledgements}

The work presented in this paper has been funded by the British Engineering and Physical Sciences Research Council (IMOOSD project, EPSRC grant No. GR/M57422).

\section{References}

1. Boehm, B, In, H, "Identifying Quality Requirements Conflicts", IEEE Software, (1996), 25-35.

2. Cheung K., Chow K., Cheung T., Consistency Analysis on Lifecycle Model and Interaction Model, Proc. of the Int. Conference on ObjectOriented Information Systems (OOIS '98), 1998, 427-441.

3. Easterbrook, S., "Handling Conflict between Domain Descriptions with Computer-Supported Negotiation", Knowledge Acquisition, Vol. 3, (1991), 255-289.

4. Emmerich W., Finkelstein, F. Montangero, C., Antonelli, S., Armitage, S., "Managing Standards Compliance". IEEE Transactions on Software Engineering, Vol. 25, No. 6, 1999.

5. Finkelstein, A., Gabbay, D., Hunter, A., Kramer, J., and Nuseibeh, B., "Inconsistency Handling In 
Draft - An updated version of this paper has appeared in the Proceedings of the $7^{\text {th }}$ International Conference on Object Oriented Information Systems

Multi-Perspective Specifications", IEEE Transactions on Software Engineering, 20, 8, (1994), 569-578.

6. Glinz M., "An Integrated Formal Model of Scenarios Based on Statecharts" Proc. of the $5^{\text {th }}$ European Software Engineering Conference, LNCS 989, Springer-Verlag, 1995, 254-271.

7. Heimdahl M.P.E, Leveson N., "Completeness and Consistency in Hierarchical State-Based Requirements", IEEE Transactions in Software Engineering, Vol. 22, No. 6, 1996, 363-377

8. Heitmeyer C., Jeffords R., Kiskis D., "Automated Consistency Checking Requirements Specifications", ACM Transactions on Software Engineering and Methodology, Vol 5, No 3, 1996, 231-261.

9. OMG, Unified Modeling Language Specification, V. 1.3a. Available from : ftp://ftp.omg.org/pub/docs/ad/99-06-08.pdf.

10.Papadimitriou C., Steiglitz K., Combinatorial Optimisation: Algorithms and Complexity, Prentice-Hall Inc., 1982.

11.Pohl K. "Process-Centred Requirements Engineering", Advanced Software Development Series, J. Kramer (ed), Research Studies Press Ltd., ISBN 0-86380-193-5, (1996), London

12. Robinson, W. and Fickas S. "Supporting MultiPerspective Requirements Engineering", In Proc. of the IEEE Conference on Requirements Engineering, IEEE Computer Society Press, Los Alamitos, CA, (1994), 206-215.

13.Si-Said S, Rolland C, Grosz G, "MENTOR: A Computer Aided Requirements Engineering Environment", In Proc. of the $8^{\text {th }}$ International Conference on Advanced Information Systems Engineering, Heraklion, Springer, (1996), 22-43

14. Spanoudakis G., and Finkelstein A. "Reconciling requirements: a method for managing interference, inconsistency and conflict", Annals of Software Engineering, Special Issue on Software Requirements Engineering, 3, (1997), 459-475.

15. Spanoudakis G., Zisman A. "Inconsistency Management in Software Engineering: Survey and Open Research Issues", Handbook of Software Engineering and Knowledge Engineering, (ed) Chang S. K., World Scientific Publishing Co., 2001 (to appear).

16. Kingman J., Taylor S., "Introduction to Measure and Probability", Cambridge Uni. Press, 1996.

17. Lamsweerde A., Darimont R., \& Letier E., "Managing Conflicts in Goal-Driven Requirements Engineering", IEEE Transactions on Software Engineering, Special Issue on Managing Inconsistency in Software Development, 1999.

18.Zisman A., Emmerich W., Finkelstein A., "Using XML to Specify Consistency Rules for Distributed Documents", $10^{\text {th }}$ Int. Workshop on Software Specification and Design, 2000.

19. Shafer G., "A Mathematical Theory of Evidence", Princeton University Press, 1976.

20.http://java.sun.com/j2se/1.3/docs/guide/awt/

21. Spanoudakis G.: "An Algorithm for Detecting Overlaps between Models of Object Interactions", Technical Report Series, TR2000/03, ISSN 1364-4009, Department of Computing, City University, November 2000.

22. Spanoudakis G., Kim H.: "Evidential Management of Inconsistencies in Object Interaction Models", Technical Report Series, TR-2000/04, ISSN 1364-4009, Department of Computing, City University, December 2000.

23. Jacobson I., Christerson M., Jonsson P., and Overgaard G..: "Object Oriented Software Engineering: A Use Case Driven Approach". Addison-Wesley, Reading, Massachusetts, 1992 